\title{
Chemoprevention based on green natural compounds
}

\author{
Capek I \\ Slovak Academy of Sciences, Institute of Measurement Sciences, Bratislava, Slovakia
}

\begin{abstract}
Plant medicine ais used in the prevention and treatment of various diseases. This approach utilizes derivatives of natural compounds such as curcumins, resveratrol, green tea extract, luteolin, soybeans, pomegranate, etc. Natural products have also been allied their ability against tumor action to trigger cell death pathways and their associated approaches. Plant products stop or exhibit inhibition of carcinogenesis at initiation or at promotion/progression step. Among them polyphenols possess many health benefits, including being potent analgesic, antibacterial, antiviral, antiparasitic, antioxidant, and anti-inflammatory agent.
\end{abstract}

The evolution of the plant-based medicine systems started from ca. 50,000 years ago by the Sumerians, Iraqes., Chinese, Tibetan, etc. [1]. From 6000 years ago through 3000 to 1500 years ago, the ancient Egyptians and ancient Greece have invented numerous curing materials obtained from natural resources. Herbs, spices, vegetables were used in food preparation as a response to the threat of food-borne pathogens. Historically, flowering plants were the source of most plant medicines [2]. They reflect their ability to produce curing agents with a broad range of pharmacological properties, including anticancer activity. Phenolic compounds, such as flavonoids, are the most promising plant-derived metabolites for the treatment of cancer [3]. Natural products have also been allied their ability against tumor action to trigger cell death pathways and their associated approaches. Apoptosis or programmed cell death plays a pivotal role in maintaining tissue homeostasis that is highly conserved among different species [4]. A hallmark of human cancers is evasion of apoptosis [5].

Modern science has proved the role of plants in the treatment of diseases, and this has been evident through the incorporation of modern drug therapy for many of the drugs of plant origin which have already been used for thousands of years by the ancient civilizations. Greek and Roman therapeutic practices were preserved through the writings of Hippocrates, especially Galen (e.g., Therapeutics), which were also as the headwaters for western medicine later [6].

Due to the side effects of synthetic drugs, lack of new pharmaceutical remedies for microbial resistance and many chronic diseases, the medicinal plants curing model appeared in medicinal research and development. Additionally, the high cost of medicines and the inability of many developing countries to purchase modern drugs have forced them to search for products in the form of medicinal plants that are proved to be cheap, efficient, safe, and culturally acceptable [7].

Green prevention model describes inhibition of carcinogenesis at initiation phase or at promotion/progression stage via the utilization of derivatives of natural compounds such as curcumins, resveratrol, green tea extract, luteolin, soybeans, pomegranate, etc. It exhibits the cell cycle inhibition and apoptosis induction, affects cancer cell growth and development (growth reduction even up to 50\%), as well as an inhibition of metastases formation, prolonging patient's survival. All these aforementioned plant products have antioxidant properties secondary to the inherent polyphenols present in them [8]. Polyphenol is a subgroup of phytochemicals which inhibit cancer development through different strategies, constrain the intracellular oncoprotein signaling along with the PKC/RAS/MPK or p13-kinase/AKT pathways, downregulate the pro-proliferative anti-apoptotic transcription factors NF- $₫ \mathrm{~B}$ and AP-1, and upregulate the carcinogen-detoxifying enzymes and DNA repair proteins [9]. Different studies show that drinking of green tea delays cancer onset between 4 and 7 years. Green tea is found to have potent antioxidants called catechins. The catechins are four types and recognized to be the active component to prevent carcinogenesis [10].

Dietary polyphenols have demonstrated significant preventive and therapeutic activities in various cancerous cells such as breast, liver, lung, prostate and skin [11]. Curcumin (Curc), a low-molecular-weight lipophilic compound [12], a major yellow pigment, is generally obtained from the rhi-zomes of Curcuma longa L. (Zingiberaceae). It absorbs in the visible region and gives fluorescence with a low quantum yield [13]. The hydrophobic Cur is a pleiotropic drug which can identify various targets and selectively penetrate through the cancerous cell membrane, compared to normal types, and initiates tumor growth prevention and apoptosis induction [14]. The application of Cur is limited due to its low solubility, poor absorption and rapid metabolism, which can be overcome in combination with a synthetized or natural carrier with a great potential for the solubility of Cur and increasing its uptake in the tumor cells and cytotoxicity.

Curcumin (diferuloylmethane) has been used widely in preclinical in vitro and in vivo models of hepatocellular carcinoma (HCC) [15]. Although the outcomes of these studies were satisfactory, the curcumin dosage used in these investigations was high due to the disadvantages of low water solubility, poor pharmacokinetics and bioavailability, and physicochemical instability [16]. The dosage of Cur used in treating xenograft models of HCC was reportedly to be $100 \mathrm{mg} / \mathrm{kg} /$ day (i.p.) for

${ }^{\star}$ Correspondence to: Ignac Capek, Slovak Academy of Sciences, Institute of Measurement Sciences, Bratislava, Slovakia, E-mail: Ignac.Capek@savba.sk

Key words: plant medicine, polyphenols, curcumin, paclitaxel, berberine, cancer

Received: September 04, 2019; Accepted: September 16, 2019; Published: September 19, 2019 
16 days [17] and $200 \mathrm{mg} / \mathrm{kg} /$ day [18], or up to $3000 \mathrm{mg} / \mathrm{kg} /$ day per os (p.o.) for 14 days [19]. Therefore, efforts have been made to overcome the limitations of the physicochemical properties of curcumin.

Curcumin is a yellow polyphenol found in turmeric (Curcuma longa) of the ginger family (Zingiberaceae). It has been part of folk medicine for centuries due to possessing many health benefits, including being potent analgesic, antibacterial, antiviral, antiparasitic, antioxidant, and anti-inflammatory agent. Curcumin has been the focus of many chemoprevention studies. Thus, it may be able to target several hallmarks of cancer [20] demonstrated a dose-dependent inhibitory effect of curcumin on colon cancer cell lines. They suggested that apoptosis and cell-cycle arrest of HCT-116 cells after curcumin treatment is mediated by caspase-3-induced cleavage of $\beta$-catenin. In addition, decreasing transactivation of $\beta$-catenin/Tcf-Lef was found to lead to the inactivation of $\mathrm{Wnt} / \beta$-catenin pathway. Moreover, treatment of HCT-116 cell line with curcumin decreased levels of c-Myc protein, a key mediator of gene regulation and differentiation that maintains self-renewal properties of embryonic stem cells. Alternatively, Ryu et al. [21] offered another mechanism of curcumin inhibitory effect on $\mathrm{Wnt} / \beta$-catenin pathway where curcumin derivatives were able to downregulate $\mathrm{p} 300$, a transcriptional coactivator of $\mathrm{Wnt} / \beta$-catenin pathway. In addition, Ryu and his group reported the suppressive effect of curcumin derivatives on $\beta$-catenin transcription that was activated by Wnt $3 \mathrm{~A}$ conditioned-medium. Furthermore, curcumin inhibits NFkB activity in ovarian, breast, head and neck, lung, and prostate cancer cell lines [22]. In the clinic, curcumin is well tolerated and has some biological activity in phase II pancreatic cancer trials [23].

Resveratrol is a further polyphenol derived from red grapes and berries. Like curcumin, a significant amount of in vitro data has shown that resveratrol inhibits the growth of multiple cancer cell lines including the breast, prostate, thyroid, head and neck, ovarian, and cervical. Resveratrol appears to regulate cancer cell growth by inhibition of IKK and suppression of NFKB activity [22]. Resveratrol also inhibits IKK activity in animal models of colitis. Data from in vivo preclinical trials shows that resveratrol can prevent tumor growth or carcinogenesis in several cancer sites including the breast, skin, prostate, gastrointestinal, and lung. Clinical trials to date, which are mostly risk assessment studies, show that resveratrol-rich products may be beneficial for cancer prevention.

Ongoing clinical trials with pure resveratrol will provide toxicity and efficacy dosage for this chemopreventive agent [24]. A third chemopreventive agent that regulates NFKB activity is epigallocatechin 3-gallate (EGCG), the major polyphenol found in green tea. In animal studies, green tea polyphenols in the drinking water resulted in a delay of primary tumor incidence and tumor burden in a mouse model of prostate cancer that correlated with a substantial reduction in NFKB activity. The data from human studies suggest that green tea polyphenols may provide greater efficacy for preventing prostate cancer than for treating cancer patients. There are multiple ongoing clinical trials to access the effects of green tea on prostate, lung, bladder, esophageal, breast, and head and neck cancer. Two other natural products known to inhibit NFkB activity, dietary isothiocyanates, from watercress, and sulforaphanes, from crucifiers, are also being evaluated in clinical trials against various cancers. Other natural products known to block NFkB activity include catechins, silymarin, caffeic acid phenethyl ester (CAPE), sanguinarine, anethole, emodin, piceatannol, capsaicin, ursolic acid, betulinic acid, flavopiridol, oleandrin [25], parthenolide, kambekaurin [22], and freeze-dried black raspberries [26].
Quercetin was intensively studied as the most abundant flavonoids in food. In vitro, quercetin inhibited the proliferation of oral cancer cell lines showing enzymatic inhibition of the thymidylate synthase, a key enzyme expressed by oral cancer cells $[27,28]$. Apoptotic properties of quercetin were also explained by the modulation of the Bax/Bcl-2 ratio in oral cancer cells. Quercetin was also associated with the prevention of oral cancer cell migration in several ways including the inhibiting of the expression of metalloprotease 2 and metalloprotease. The effect of quercetin extends beyond prevention; quercetin showed some cancer therapeutic potential. The combination of quercetin with cisplatin (a chemotherapeutic drug) induced apoptosis and decreased the cells' resistance to the chemotherapeutic medication $[27,28]$.

Active pharmaceutical ingredients (APIs) with anticancer activity are usually isolated from plants allowing them to be tested for a specific mode of action at defined concentrations without side effects caused by other metabolites. The most prominent example is paclitaxel, a taxane found in the bark of the Pacific yew tree [29]. Small-molecule anticancer compounds can also be produced in cultured plant cells [30]. Paclitaxel is used to treat ovarian, breast, and pancreatic cancers among others [29] the extraction of paclitaxel from Pacific yew trees [29] requires a large cultivation area to grow a sufficient number of trees over a period of up to 200 years. The trees must be logged and stripped of bark to extract paclitaxel, killing the trees in the process.

Myriad of many plant products exist that have shown very promising anticancer properties. The plant-natural compounds derived from Tinospora cordifolia, Ocimum sanctum, Bauhinia variegata, etc. have shown promise as anticancer agents and outlines their potential mechanism of action. The anticancerous compounds present in the Tinospora cordifolia are berberine and palmatine. Berberine is present in the stem whereas palmatine is present in both root and stem [31]. The berberine and palmatine both are protoberberine alkaloids that show anticancerous activity. Palmatine is a close structural analogue of berberine that both bear the same tetracyclic structure (7,8,13,13-tetrahydro 9,10 dimethoxy berberinium) but differ in the nature of substitutes at position 2,3 on the benzo ring, where it is dimethoxy for palmatine and methylene dioxy for berberine [32].

Free radicals, reactive oxygen species (ROS), and radiations induce the DNA damage, including the oxidation, strand breakage, and ionization which may lead to defects in the genes involved in proliferation and cell signaling pathways that are crucial for tumor growth and cancer progression, so antioxidants play a protective role in preventing cellular damage due to oxidation [33,34]. Lipoxygenase, xanthine oxidase, and cyclooxygenase (COX2) are the three important enzymes that are responsible for generating the reactive oxygen species in the cells and berberine has the ability to reduce the activity of xanthine oxidase and COX2, which finally decrease the level of reactive oxygen level in the cell. But sometime berberine increases the amount of reactive oxygen species to induce several apoptotic signaling pathways, including mitogen-activated protein kinase (MAPK), extracellular signal-regulated kinase 1/2 (ERK1/2), c-Jun N-terminal protein kinase (JNK) and Akt, as well as calciumdependent pathways. So these different effects of berberine, whether it increases or decreases the cellular reactive oxygen species content, depend upon the cell conditions and types. For example, berberine induces reactive oxygen species production in prostate cancer cells, but not in the normal epithelial cells. Berberine induces apoptosis and inhibits cell proliferation in various cell lines derived from breast, lung, colon, and liver cancer by different pathways: Fasdependent pathway and p53-dependent pathway as follows. 


\section{Conclusion}

Chemopreventive natural agents have been part of folk medicine for centuries due to possessing many health benefits, including being potent analgesic, antibacterial, antiviral, antiparasitic, antioxidant, and anti-inflammatory agent. Natural products are known to block NFkB activity include catechins, silymarin, caffeic acid phenethyl ester, sanguinarine, anethole, emodin, piceatannol, capsaicin, ursolic acid, betulinic acid, flavopiridol, oleandrin epigallocatechin 3-gallate, curcumin, quercetin, etc. Furthermore, plant medicines induce reactive oxygen species production in cancer cells, but not in the normal epithelial cells. They induce apoptosis and inhibits cell proliferation in various cell lines derived from breast, lung, colon, and liver cancer by different pathways.

\section{Glossary}

Cancer is a disease that is associated with genetic lesions and epigenetic modifications. During oncogenesis and malignant cellular transformation, DNA undergoes significant changes in methylation, resulting in both activation and silencing of genes. Particularly genes involved in cell cycle regulation, cell growth, and differentiation are affected leading to uncontrolled expansion of cancerous cells. Cancers are classified mainly in three groups, carcinomas, leukemias/ lymphomas, and sarcomas, based on their tissue origin during embryonic development. About $90 \%$ of the cancers in humans are malignancies of epithelial cells, the carcinomas. Leukemias and lymphomas arise from the hematopoietic cells of bone marrow and are responsible for roughly $8 \%$ of human cancers. Whereas leukemias tend to proliferate as single cells, lymphomas grow as aggregates of tumor cells. Sarcomas, which are relatively rare in humans (approximately $1 \%$ of total cancer cases), are solid tumors of connective tissues.

\section{References}

1. Mamedov N (2012) Medicinal plants studies: history, challenges and prospective. Med Aromat Plants 1: e133.

2. Fabricant DS, Farnsworth NR (2001) The value of plants used in traditional medicine for drug discovery. Environ Health Perspect 109: 69-75.

3. Asensi M, Ortega A, Mena S, Feddi F, Estrela JM (2011) Natural polyphenols in cancer therapy. Crit Rev Clin Lab Sci 48: 197-216.

4. Evan GI, Vousden KH (2001) Proliferation, cell cycle and apoptosis in cancer. Nature 411: $342-348$

5. Hanahan D, Weinberg RA (2000) The hallmarks of cancer. Cell 100: 57-70.

6. Robson B, Baek OK (2009) The engines of hippocrates: from the dawn of medicine to medical and pharmaceutical informatics. John Wiley \& Sons, 2009 Hoboken, NJ.

7. Saba NF, Haigentz M Jr, Vermorken JB, Strojan P, Bossi P, et al. (2015) Prevention of head and neck squamous cell carcinoma: removing the "chemo" from "chemoprevention". Oral Oncol 51: 112-118.

8. Sheth SH, Johnson DE, Kensler TW, Bauman JE (2015) Chemoprevention targets for tobacco-related head and neck cancer: past lessons and future directions. Oral Oncol 51: $557-564$.

9. Fujiki H, Imai K, Nakachi K, Shimizu M, Moriwaki H, et al. (2012) Challenging the effectiveness of green tea in primary and tertiary cancer prevention. $J$ Cancer Res Clin Oncol 138: 1259-1270.

10. Lall RK, Syed DN, Adhami VM, Khan MI, Mukhtar H, et al. (2015) Dietary polyphenols in prevention and treatment of prostate cancer. Int J Mol Sci 16: 3350-3376.

11. Sahu A, Kasoju N, Bora U (2008) Fluorescence study of the curcumin-casein micellen complexation and its application as a drug nanocarrier to cancer cells. Biomacromol 9: $2905-2912$.

12. Patra D, Baraka C (2011) Synchronous fluorescence spectroscopic study of solvatochromic curcumin dye: spectrochim. Acta A Mol Biomol Spectrosc 79: 10341041.
13. Chaudhary LR, Hruska KA (2003) Inhibition of cell survival signal protein kinase B/ Akt by curcumin in human prostate cancer cells. J Cell Biochem 89: 1-5.

14. Darvesh AS, Aggarwal BB, Bishayee A (2012) Curcumin and liver cancer: A review. Curr Pharm Biotechnol 13: 218-228.

15. Yallapu MM, Nagesh PK, Jaggi M, Chauhan SC (2015) Therapeutic applications of curcumin nanoformulations. AAPS J 17: 1341-1356.

16. Ning L, Wentworth L, Chen H, Weber SM (2009) Down-regulation of Notch1 signaling inhibits tumor growth in human hepatocellular carcinoma. Am J Transl Res 1: 358-366.

17. Cui SX, Qu XJ, Xie YY, Zhou L, Nakata M, et al. (2006) Curcumin inhibits telomerase activity in human cancer cell lines. Int J Mol Med 18: 227-321.

18. Yoysungnoen P, Wirachwong P, Bhattarakosol P, Niimi H, Patumraj S (2005) Antiangiogenic activity of curcumin in hepatocellular carcinoma cells implanted nude mice. Clin Hemorheol Microcirc 33: 127-135.

19. Jaiswal AS, Marlow BP, Gupta N, Narayan S (2002) Beta-catenin-mediated transactivation and cell-cell adhesion pathways are important in curcumin (diferuylmethane)-induced growth arrest and apoptosis in colon cancer cells. Oncogene 21: 8414-8427.

20. Ryu MJ, Cho M, Song JY (2008) Natural derivatives of curcumin attenuate the Wnt $/ \beta$ catenin pathway through down-regulation of the transcriptional coactivator p300. Biochem Biophys Res Commun 377: 1304-1308.

21. Brown M, Cohen J, Arun P, Chen Z, VanWaes C (2008) NF-kappaB in carcinoma therapy and prevention. Expert Opin Ther Targets 12: 1109-1122.

22. Dhillon N, Aggarwal BB, Newman RA, Wolff RA, Kunnumakkara AB, et al. (2008) Phase II trial of curcumin in patients with advanced pancreatic cancer. Clin Cancer Res 14: 4491-4499.

23. Bishayee A (2009) Cancer prevention and treatment with resveratrol: from rodent studies to clinical trials. Cancer Prev Res (Philadelphia, Pa) 2: 409-418.

24. Dorai T, Aggarwal BB (2004) Role of chemopreventive agents in cancer therapy. Cancer Lett 215: 129-140.

25. Huang C, Huang Y, Li J, Hu W, Aziz R, et al. (2002) Inhibition of benzo(a)pyrene diol-epoxide-induced transactivation of activated protein 1 and nuclear factor kappaB by black raspberry extracts. Cancer Res 23: 6857-6863.

26. Maggioni D (2015) Flavonoids in oral cancer prevention and therapy. Eur J Cancer Prev 24: $517-526$

27. Chen S-F (2012) Quercetin suppresses drug-resistant spheres via the p38 MAPKHsp27 apoptotic pathway in oral cancer cells. PLoS One 7: e49275.

28. Wani MC, Horwitz SB (2014) Nature as a remarkable chemist: a personal story of the discovery and development of Taxol. Anticancer Drugs 25: 482-487.

29. Tabata H (2004) Paclitaxel production by plant-cell-culture technology. Adv Biochem Eng Biotechnol 87: 1-23.

30. Mittal J, Sharma MM, Batra A (2014) Tinospora cordifolia: a multipurpose medicinal plant-A review. J Med Plant Stud 2: 32-47.

31. Bhadra K, Maiti M, Kumar GS (2007) Molecular recognition of DNA by smal molecules: AT base pair specific intercalative binding of cytotoxic plant alkaloid palmatine. Biochim Biophys Acta 1770: 1071-1080.

32. Tanno T, Matsui W (2011) Development and maintenance of cancer stem cells under chronic inflammation. J Nippon Med Sch 78: 138-145.

33. Cooper G (2000) The cell: A molecular approach. Sinauer Associates Inc 2000, Sunderland, MA.

34. Goldsby RA, Kindt TJ, Kube J, Osborne BA (2002) Immunology. Freeman WH \& Company, 2002, New York, NY.

Copyright: (C2019 Capek I. This is an open-access article distributed under the terms of the Creative Commons Attribution License, which permits unrestricted use, distribution, and reproduction in any medium, provided the original author and source are credited. 